\title{
Über das Lob in der Kunstgeschichte und sein Schicksal
}

\author{
Fehl, Philipp
}

Veröffentlicht in:

Abhandlungen der Braunschweigischen Wissenschaftlichen Gesellschaft Band 48, 1997, S.251-269

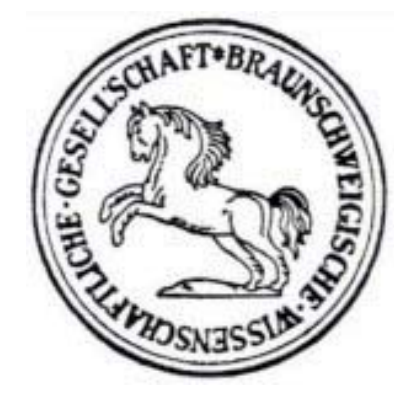

Verlag Erich Goltze KG, Göttingen 


\title{
Über das Lob in der Kunstgeschichte und sein Schicksal*
}

\author{
Von Philipp Fehl**, Vatikanstadt \\ (Eingegangen am 14.11.1997)
}

\begin{abstract}
In memoriam
meiner unvergeßlichen Braunschweiger Freunde

Edgar Rosen und Martin Gosebruch

und ihrer Anliegen als Historiker und Menschen. ${ }^{1}$
\end{abstract}

Das Lob bei Festen der Erinnerung ist immer am richtigen Platze, ist es doch der einzig mögliche Ausdruck des Glücks, der Dankbarkeit, der Anerkennung für geleistete Dienste, für das gegebene Vorbild, für erwiesene Menschlichkeit, für die Übertragung von Generation zu Generation eines großen nachahmungswürdigen Beispiels das wir feiern, in der Hoffnung uns auch selbst daran zu bilden und erbauen. Es ist das Setzen eines Denkmals, einer der schönsten Aufgaben der Kunst die leider jetzt nicht oft gewürdigt wird, weil man glaubt, daß Denkmäler, die ja ihrer Natur entsprechend, rühmliches über den Gegenstand ihrer Verherrlichung aussagen, lügen. Doch dem ist nicht so. Sie rühmen ja nur was sie zeigen und lügen oder betrügen nur dann wenn das, was sie zeigen nicht schön und lobenswert, sondern nur eitel und keck oder gar unmenschlich ist, auch dann wenn die Künstler und ihr Publikum eben gerade das Unmenschliche für lobenswert halten und das Häßliche, die Härte, für Tapferkeit ausgeben. Es mag ja sein, da $\beta$ sie es nicht besser wissen und wir müssen alle in Demut einsehen, daß wir, wenn wir Werturteile fällen, uns irren können, ja zum unweigerlichen Irrtum verurteilt wären, wenn uns nicht, aus dem großen Schatz der Vergangenheit, Kunst und Geschichte Vorbilder zeigten, die uns besser begreifen lassen, was die Kultur des verdienten Ruhmes und der Liebe uns zu bieten hat und wir erkennen, wie sehr wir diesem historischen Betrachten das, wenn wir einmal mit ihm angefangen haben, uns nicht mehr los läßt, für die Gestaltung unseres eigenen Lebens in Dankbarkeit verpflichtet sind.

\section{Alte Voraussetzungen}

Die Geschichte des Lobs, des wahren Lobs der Kunst, ist so alt wie die Schöpfung selbst. ,Gott sprach, es werde Licht. Und es ward Licht und Gott sah, daß das Licht gut war." Sechs Tage lang schuf Gott die Welt und prüfte sein Werk und lobte es, es war gut. Die Engel aber, als sie das Werk betrachteten, jubelten ihr Lob Gottes der die Welt

\footnotetext{
* Nach einem Vortrag in der Klasse für Geisteswissenschaften am 8.11.1996.

1 Eine erweiterte Fassung dieses Vortrages erscheint demnächst in dem Band: Philipp Fehl, Kunst und Menschlichkeit, Böhlau Verlag/Wien.
}

** Prof. em. Dr. phil. Ph. Fehl - The Cicognara Program - Biblioteca Apostolica Vaticana . I-00120 Vatikanstadt 
so gut erschuf. Wir hören ihren Gesang in Haydn's Schöpfung und jubeln im Geiste mit, so schön und so alle Welt erfüllend ist ihre reine Freude im Himmel und so glückbringend.

Mein Versuch, das Schicksal des Lobes in der Kunstgeschichte zu betrachten, besteht aus zwei sehr ungleichen Abschnitten in historischer Folge; der Zeit in der das Lob in der Kunst wie in der Kunstgeschichte sozusagen das Herz des Erlebens war, und der Zeit der letzten hundertundzwanzig Jahre, die in der Kunstgeschichte vom akademischen Betrieb geprägt wird und sich schon daher des Lobes als laienhaft gerne enthält, und in der die Kunst viel komplizierter wenn auch nicht unbedingt besser über sich selbst und ihre Geschichte und die Gesellschaftsformen, an denen sie Lob oder Tadel übt, laut in ihren Werken nachdenkt.

Doch lassen sich die zwei Stile der Kunstgeschichte nicht so einfach von einander unterscheiden, wie ein chronologisch geordneter Überblick es uns nahelegt. Hundertundzwanzig Jahre lassen sich so leicht nicht auf einen gemeinsamen Nenner bringen. Die Widersprüche von Lob und Tadel und der oft genug sich selbst lobenden Enthaltung von Werturteilen, ja die Geisteshaltung selbst, die gleichzeitig und oft nebeneinander an den selben Fakultäten in den merkwürdigten, Staunen erregenden Gegensätzen existierten und existieren, mahnen uns zur Vorsicht, wenn wir uns versucht fühlen, von einer sogenannten Vorgeschichte der Kunstgeschichte und einer sie überragenden wirklichen, also zünftigen Kunstgeschichtsschreibung reden zu wollen. Vieles das ich im ersten Abschnitt des Versuches behandle, wirkt, wenn auch getriibt durch die Verhältnisse der Zeit, im zweiten Teil unvermindert weiter fort; vieles, weil ja schon alles einmal dagewesen ist worauf es beim Denken ankommt, ist so neu nicht, wie man leicht glaubt, entspricht doch schon die Verteidigung, mit der Adam und Eva Gott und sich selbst den Sündenfall 7u erklären versuchten, den Voraussetzungen der Methodik einer modernen Geschichtswissenschaft und Psychologie, die die Taten der Menschen, die sie erforscht, aus den Bedingungen der Umwelt ableiten und die handelnden Figuren der Geschichte dementsprechend der Verantwortung für ihre Handlungen entheben:

„Darauf fragte er [Gott]: ,Wer hat dir gesagt, daß du nackt bist? Hast du von dem Baum gegessen, von dem zu essen ich dir verboten habe?‘ Adam antwortete: ,Die Frau die Du mir beigesellt hast, sie hat mir von dem Baum gegeben, und so habe ich gegessen." "

,.Gott der Herr sprach zu der Frau: ,Was hast du getan. ‘ Die Frau antwortete: ,Die Schlange hat mich verführt, und so habe ich gegessen." “

Gott hat, wie wir alle nur zu gut wissen, diese Erklärungen nicht anerkannt. Er glaubt an den freien Willen der Menschen. Er hat ihn uns ja gegeben. Bis zum heutigen Tag gibt es Historiker, auf die die Geschichte vom Sündenfall, auch wenn sie nicht unbedingt daran glauben, einen Eindruck macht. Manche von diesen enthalten sich dennoch des Lobes und des Tadels aus Gründen der Bescheidenheit und des guten Geschmacks. Eine andere Fraktion aber hält Werturteile, als Lob und Tadel, für unwissenschaftliche Einmischungen in den Lauf der Geschichte, die längst von der Wissenschaft überholt worden sind und relativiert wurden, sogar die Lehre des Sündenfalls und der ihm entsprechenden 
Verkündigung des Jüngsten Gerichts, in der Bibel sowie in den Werken der Kunst, die im Stande sind, uns das Ende der Zeiten, der Zukunft voraus, unserer Gegenwart erschütternd vor die Augen und die Seele zu bringen.

Das Lob der Kunst in seiner poetischen Form, das uns die poetische Leistung des Künstlers beschreibend vorstellt und erklärt, verdanken wir der Antike. Wie Pallas Athene aus dem Kopfe des Zeus, springt es vor uns vollendet auf in Homers Beschreibung des Schildes, welches Hephaistos für Achilles schmiedete und schmückte. Nur ein Gott konnte es erschaffen, aber wir alle können es vor Augen sehen, wenn wir Homers Worten lauschen und dabei der Versuchung widerstehen, den Gesang auf seine mögliche archaeologische Nutzbarkeit zu prüfen. Das Schild glänzt und bewegt sich und die auf ihm dargestellten Historien, die den Lauf der ganzen Welt umfassen, erwachen in der Bewegung zum Leben. In der Beschreibung, die selbst zum Leben wird, erweist sich das Lob des Kunstwerks. Dabei wird auch, wohl zum ersten Male, die Kunstgeschichte in die Literatur eingeführt:

Ferner schuf er darauf einen Reigen, der rühmliche Künstler,

Jenem ähnlich, den einstens in Knosos, der weiten Feste,

Daidalos hatte gefügt für die lockige Maid Ariadne.

Blühende Jünglinge dort und reichbegüterte Mädchen

Tanzten den Reigen und hielten einander gefaßt an den Händen.

Duftige Schleier trugen die Mädchen und Hemden die Knaben,

Schöngewebt und sanft erglänzend von schimmerndem Öle.

....

Dicht aber stand das Volk um den reizenden Reigen versammelt,

Froh sich ergötzend; und unter der Menge ein göttlicher Sänger

Rührte die Saiten und sang, indes zwei springende Gaukler

Räder schlugen, sobald er zu singen begann, in der Mitte.

Endlich schuf er darauf die Gewalt des Okeanosstromes

Rings um den äußersten Rand des festgebildeten Schildes.

Der Dichter läßt uns nie ganz vergessen, daß wir einer Illusion gegenüberstehen, nämlich der, die der göttliche Künstler erschaffen hat, er führt uns aber virtuos an die Grenze derselben, die an die Wirklichkeit anschließt, und wir vergessen fast schon im Anblick des Schildes, daß wir es in einem Gedicht zu sehen bekommen, das ein Sänger, Homer selbst, vorträgt, der uns einen Sänger im Schilde vorstellt, der selbst singt. Das Wunder der Illusion, die er uns bringt, ist nicht, daß die Kunst uns täuschen kann - das wäre ein Gaukelspiel der Delusion - sondern, daß sie zur Wahrheit wird gerade weil wir wissen, daß es der Kunst bedarf, der Kunst, die der Dichter eben deshalb lobt, um dem Bild die Sprache, die Bewegung, die Wahrheit der Natur selbst zu verleihen. Homers kunstgeschichtlicher Exkurs, der Daedalus rühmt, ist nicht nur schmückendes Beiwerk. Er erinnert uns daran, daß Daedalus seinen Gestalten wirkliche Lebensnähe verleihen konnte, indem er sie, so wie Automaten, beweglich bildete. So wenigstens ging die Sage. Hephaistos, der Gott, war ein noch viel besserer Künstler als der findige und begabte Urkünstler, der die Bewegung der Natur wiederholen konnte. Der Gott machte sein Kunstwerk zur Natur, weil er der Mechanik entsagte, er wendet sich, wie Homer 
selbst, der ihn hier in seinem Gesange nachahmt, an unsere Vorstellungskraft und so an unsere Seele.

Der Erfolg des Gesangs vom Schild des Achilles befruchtete die Kunst der Poesie, in Lied und Bild, von der Zeit Homers an in zahllosen Variationen und Überlieferungen, bis in die Zeit Goethes und darüber hinaus. Ovids bildhafte Darstellung von Bildern in seinen Metamorphosen wäre undenkbar ohne das Vorbild Homers und die Bilder von Tizian, Rubens, und Tiepolo, um nur die freiesten Wiederhersteller des gesungenen Bildes im Bilde zu nennen, wären ungemalt geblieben. Vergil stellte sich selbst, mit eigener Hand, sozusagen, ein kostbares Schild für Aeneas her, noch besser als das von Hephaistos, denn seine Bilder auf dem Schild ahmen nicht nur die Natur nach, sie sagen auch die Zukunft voraus. Das Beispiel hat sowohl Dichter wie Maler dazu angespornt, Zukunft und Gegenwart in einem Bilde miteinander so zu verbinden, daß sie sich gleichsam umarmen im Widerspiel der Zeiten.

Das große Vermächtnis Vergils an die Kunst und Kunstgeschichte ist seine Beschreibung der Bilder in der Vorhalle des Tempels der den Trojanischen Flüchtlingen feindseligen Juno in dem von Dido neugegründeten Karthago. Aeneas und sein Freund Achates, aus Vorsicht von Venus in eine Wolke eingehüllt, so daß sie andern unsichtbar, die vermutlich barbarische Gegend in relativer Sicherheit auskundschaften können, stehen nun vor der Vorhalle dieses Tempels und werden staunend gewahr, daß die Bilder, in feiner, lebenswahrer Arbeit, die Geschichte ihres eigenen Unglücks darstellen. Aeneas sieht den Kampf vor der Stadt, die nicht mehr steht, er sieht Priamus auf den Knien vor Achilles, er sieht den toten Hector und seine Stimme und die des Dichters bricht; der Dichter spricht die Zeile nicht zu Ende. „Oh welches Land, mein Achates,“ sagt Aeneas, „,kennt jetzt nicht unser Leid? Auch hier [in diesem Land] gibt es das Lob der Tugend. Auch hier werden Opfer des Unglücks beweint. Fürchte nicht länger; die Kunde unseres Schicksals wird auch Dir in mancher Hinsicht Rettung bringen."

Dieses Lob der Kunst, die vermag, fremdes Leid mit Mitleid darzustellen, darf als Voraussetzung betrachtet werden für die große Versöhnung des Abbildungsverbotes der Bibel - da die Kunst notorisch unverläßlich ist und, wenn der Künstler sich nicht prüft, sie jeder Eitelkeit und jedem Götzendienste zum Handlanger wird - mit der Kunst der Antike in ihrem Fortleben im christlichen Abendland. Mitleid, die Übung teilnehmenden Erlebens durch die Kunst, die Erkenntnis des Leidens und auch der Größe der Fremden im Unglück in der Erschütterung des eigenen Herzens ist die Voraussetzung für die Erfahrung der Gottesfurcht, die der Anfang der Weisheit ist.

Wir finden die Bilder des Tempels der Juno wieder in neuer Einkleidung in Dantes Purgatorio, wo die Kunst den reuigen aber durch Buße noch zu reinigenden Sündern ihre milde, herzbewegende Hilfe spendet und wir sehen und erleben eben diese Kunst im Relief gegossen auf den großen Bronzetüren Ghibertis an dem Baptisterium in Florenz. Nicht umsonst erzählt die Legende, Michelangelo hätte vor diesen Türflügeln ausgerufen, sie wären die Pforten des Paradieses.

Und so wie die Bilder Homers und dann Vergils, so befruchteten die vielen Bilder, die Ovids Metamorphosen uns bieten, die poetisch-bildnerische Vorstellungskraft 
der Künstler des Mittelalters und der Neuzeit, wenn auch selten mehr bis in unsere neueste Neuzeit.

Die für das Weiterleben der Künste vielleicht fruchtbringendste Gestalt der Antike, heute oft vergessen, war durch die ganze Renaissance hindurch, bis zu Goethe und Heinrich Brunn, Philostratus der Ältere, der im frühen dritten Jahrhundert nach Christi Geburt die Bilderklärung und Bildbetrachtung in öffentlichen Reden lehrte, und was er vorstellte, zum bildhaften Erlebnis machte. Er wird jetzt, soweit man sich noch mit ihm befaßt, eher streng beurteilt, denn er hatte das Unglück, in was wir im Nachhinein eine Spätzeit der Antike nennen, zu leben. Man glaubt also, man könne ihm nicht trauen, wenn er über Bilder spricht, die einer andern, älteren Zeit angehören. Er kann sie nur, wenn er sie nicht überhaupt erfunden hat, im Sinne seiner Zeit begreifen. Philostratus lobt auch zu viel für den Geschmack der modernen Archaeologie und sagt ihr nicht, wie die Bilder eigentlich ausschauten. Er läßt uns die Bilder erleben, oft in der Sprache, die wir in Homers Darstellung des Schilds des Achilles bewundern, oft in derjenigen der Dichter, aus deren Werken der Maler sein Thema entnahm, aber das Erlebnis, das sich immer auf die Darstellung der lebendigen Natur bezieht, gestattet uns nicht, ich möchte fast sagen, Gott sei Dank, stilkritische Analysen an ihm zu versuchen.

Wir wandern mit Philostratus durch eine Villa, deren Logen - ich stelle mir sie so vor wie Raphaels Logge des Vatikanischen Palasts - reichlich auf drei oder vier Stöcken mit Bildern geschmückt sind. Junge Herren kommen und bitten Philostratus, mit ihnen durch die Galerien zu gehen und die Bilder zu besprechen. Doch Philostratus weigert sich, bis endlich der junge Sohn des Hauses, ein etwa zehnjähriger Knabe, sich den Bitten anschließt. „Gehe du mit mir,“ sagt Philostratus endlich, „und wir werden uns die Bilder gemeinsam anschauen. Ihr aber,“ sagt er zu den jungen Männern, ,folget uns nach und wenn ihr Fragen zu stellen habt, fraget.“

Und so, im Gespräch, in dem vorzüglich Philostratus redet, erschließen sich die Bilder unserem geistigen Auge und werden wesentlich. Man hört die Leute im Bilde singen, die Andrier werden trunken, wir sehen, wie sie tanzen und erfahren, wie im Wort ihre Zunge leicht anstößt, nein, wir hören es und singen fast mit. Äpfel, welche die Eroten einander zuwerfen, leuchten nicht nur rot auf, wir verspüren fast ihren Geruch, der Duft des Liebeshains ist himmlisch, und siehst Du nicht dort, in der Grotte, in die wir gar nicht hineinschauen können, ist die Statue der Venus; nein es ist die Göttin selbst und die Nymphen, die dort wohnen, bringen ihr ihre Gaben.

Raphael, Tizian, Rubens und viele andere haben diese Bilder des Philostratus gemalt, sie wollen wohl selbst so besprochen werden, in der Tradition der erlebenden Beschreibung, die sie begeisterte, als sie sich die Aufgabe stellten, eine solche Kunst, die wie die Antike im Worte zu uns spricht, im Bilde wieder aufleben zu lassen.

\section{Vasari und Michelangelos «Jüngstes Gericht»}

Vasari, und in seiner Folge die ganze Kunstgeschichte der Renaissance, bis hinauf in das frühe 19. Jahrhundert, lebten und fanden ihr métier und ihre Aufgabe in dem Vorbilde der verlebendigenden, die Natur wiedererschaffenden Darstellungsfähigkeit der 
Dichtkunst und der Rhetorik. Ihre Schutzpatrone waren Homer, Vergil, Ovid, Cicero, Quintilian, Philostratus, und, in Vergils christlicher Nachfolge, Dante. Die Kunst und Forschung der lobenden Kunstgeschichtsbeschreibung betrieb ihr Geschäft mit einer schönen Selbstverständlichkeit, die über ihre „Grundbegriffe" nicht nachdenken mußte. Lebte und wirkte sie doch in einer Tradition, in der die Kunstwerke, die sie betrachtete, der selben poetisch erfaßten Sprache des Lobs und seiner Erkenntnisfähigkeiten entsprangen wie die der Liebe zur Kunst, welche die Kunsthistoriker, die oft selbst Künstler von Bedeutung waren, vermitteln und loben wollten.

Um zu erfassen, was sie konnten und suchten, wenden wir uns dem Höhepunkt von Vasaris lange in seinen ernsten Belangen vorbildlichem Buche der Vite dei più eccellenti pittori, scultori, e architetti zu. Es ist seine rühmende Beschreibung des «Jüngsten Gerichts» von Michelangelo, in der er alles, was er uns über die Würde der Kunst und ihre Fähigkeit, uns zur Besinnung auf unsere Menschlichkeit zu erheben, vorausgebildet und inn selbst im Erlebnis des Kunstwerkes, im Anblicke wie in der Erinnerung, immer von neuem bildend, beinhaltet findet.

Wenn wir uns nun dem Bilde zuwenden, so sehen wir, wie es bei einer lebensnahen, ja zum Leben und Erleben drängenden und lebendig werdenden Darstellung eines solchen Themas gar nicht anders sein kann, ein Werk, das den höchsten Anspruch an unsere moralische Urteilskraft stellt. Vor diesem Richter prüfen wir nicht das Bild, sondern uns selbst.

Es gibt wohl in der ganzen Geschichte der Kunst kein moralisch anspruchsvolleres Bild als dieses «Jüngste Gericht». Es zieht daher, von der Zeit seines Ursprungs her bis vor über hundert Jahren, als die damals neu entdeckte Kunstwissenschaft begann, auf kühle beobachtende, wertfreie Analysen als Voraussetzung für die Beurteilung von Kunstwerken zu drängen, eine lebhafte moralisch fundierte, argumentreiche, einerseits begeisterungsfrohe, anderseits bis zur Entrüstung ablehnende, ununterbrochene Debatte um das Verstehen dieses Kunstwerks als Erleuchtung oder Verdunkelung der göttlichen Größe, Liehe, der Gerichtsamkeit selbst und, im Zusammenhang mit ihr, der Schicklichkeit nach sich.

Wir sind an ein solches Betrachten von Kunstwerken nicht mehr gewöhnt, auch wenn es sich um ein Jüngstes Gericht handelt, das doch unser Wissen um Schuld und Sühne und die Hoffnung auf Vergebung unserer Sünden in allen seinen Voraussetzungen in einem gewissen Sinne direkt anspricht. Wir kennen es schon zu gut als ein zeitbedingtes Denkmal der Kunstgeschichte, um es auch wirklich noch zu erleben. Es wird uns von Fachgelehrten, die schon wissen, was da zu sehen ist, mit Hilfe von Dias gezeigt, bevor wir, in der Einsamkeit unseres eigenen Gewissens, ihm als Privatmenschen, sozusagen im Zivil, gegenübertreten können. Sehen wir es dann auch im Original in der Sistina, so sind wir oft versucht, eben dann nur das zu agnoszieren, was wir im Schulbetrieb lernten oder auch, unter Umständen, lehren.

Wie kommt man davon los und einigermaßen wieder zu sich selbst, um zu dem Kunstwerk ein tugendhaftes Verhältnis zu gewinnen, ob man nun mit ihm gehen will oder auch, nach bestem Gewissen und mit der nötigen Achtung und Wertschätzung, die ein Künstler wie Michelangelo auf alle Fälle verdient, uns - wie es seiner Zeit Jakob Burckhardt erwählte - dagegen zu verwahren? Kunstwerke sind ja nicht nur eine Er- 
leichterung des Lebens und gütige Gestalter und Erweiterer unserer Vorstellungskraft, sondern auch mögliche Verführer derselben. Die Kunst wirkt hier wie die Rhetorik, die uns vieles zu lehren vermag und unser Verständnis der tiefsten Dinge läutert, die aber auch, wenn wir uns für einen Redner begeistern und nicht aufmerksam darauf sind, was ihn eigentlich dazu bewegt, die imposanten Maschinen seiner Kunst spielen zu lassen, uns blenden und in Schuld verstricken kann. All dies verbürgt uns die Antike. Denken wir nur an die Reden, die Thukydides erfand, um an Hand des Spiels dieser Rhetorik, die nur überzeugen aber nicht nachdenklich sein wollte, die Verstrickung Athens in immer größeres Unglück und Schuld sich und seinen Lesern zu erklären. Die Kunst, sowohl wie die Kunstkritik der Renaissance, erwachte aus diesem Wissen um das Janushaupt der Redekunst und es genügt hier wohl die Zeugenschaft Leone Battista Albertis anzurufen, um daran zu erinnern, mit welcher moralisch fundierten Hoffnung die Kunst ihre hohen und höchste Ziele steckte, in freier und sich immer wieder von Neuem läuternder Rede.

Den besten Zugang zu dem Leben des «Jüngsten Gerichts» Michelangelos bietet uns die begeisterte und beglückte Beschreibung des Bildes von Vasari. Wie er uns erzählt, was das Bild darstellt, erlebt er auch den Bildgehalt und wir erleben ihn mit ihm.Viele seiner ursprünglichen Leser kannten das Bild nur aus seiner Beschreibung, höchstens dabei unterstützt von einigen schon von der Natur der Reproduktion her bedingten unzulänglichen Kupferstichen. Wer dieses Werk der bildbeschreibenden Poesie, die im Stande ist, sich mit dem, was sie beschreibt, zu identifiziert und selbst berührt ist von dem Mitleid und der Furcht und der Gottessehnsucht, die sie in uns erwecken will, weil sie sie im Bilde erkennt, unvoreingenommen liest, wird von Sehnsucht ergriffen, das Bild selbst zu sehen und will nach Rom pilgern, um dort vor dem Bilde zu sehen, wie sehr die Kunst imstande ist, über die Kunst hinaus zu deuten in die Gegenwart des Weltendes und des göttlichen Urteils mit allen seinen Schrecken und Erlösungen, als ob der Künstler dabei gewesen wäre bei dem, was noch nicht geschehen ist.

Vasaris erweckende Beschreibung beginnt mit einem Dante Zitat. Die Energie, die Lebenskraft, die Michelangelo seinen Figuren verlieh, erweckten Dantes Worte ,morti li morti, i vivi paresan vivi (die Toten sind wirklich tot, und die Lebenden scheinen zu leben) [ Michelangelo ] ha verificato il detto di Dante." Das Zitat bezieht sich auf eine Stelle im Purgatorio, in der Sündige, die im Leben stolz waren und die Sünde noch zu bereuen vermochten bevor sie starben, ihre Schuld abbüssen und überwinden, und Gott hilft ihnen dabei mit Hilfe der Kunst. Als Reliefs in Stein geschnitten sehen sie dort, während sie von ihnen gebeugt schwere Lasten tragen, Szenen aus der Geschichte, die zeigen, welch Unrecht der Stolz schafft und wie herrlich und süß die Demut ist. Seufzend erleben die Sünder die Wahrheit der göttlichen Kunst und ihre Tränen bessern sie so wie sich Dante bessert und gereinigt wird, da er ihre Reue sieht. Nun aber, meint Vasari, Dank der Kunst Michelangelos, können wir wirklich vor uns sehen, was Dante nur beschreiben konnte; hier zeigt uns Michelangelo sogar noch mehr als Leben und Tod; wir erkennen, wir begreifen das Elend der Verdammtem und das Glück der Seligen, "Quivi si conosce la miseria dei dannati e l'allegrezza de'beati.“

Der Text der Bildbeschreibung, die folgt, führt nur aus, in beschreibenden Worten, was diese Titelvignette, mit der er sie einführt, wie ein Emblem in sich enthält. Die Fähigkeit des Künstlers, der nachgeahmten Natur Leben zu verleihen und die poetisch-sitt- 
liche Läuterung, die er sucht und uns vermitteln will, sind innigst miteinander verbunden, wie - im Leben - die Seele mit dem Leib. Vasaris Beschreibung überstürzt sich in der Vermengung der beiden. Hier, in bunter Folge, der Anblick von Heil und Verdammung, Auferstehung der Toten, das Gedröhne der Trompeten und da die Kunst der Zeichnung, die gelungenen Verkürzungen, das Schweben und das Fallen der Seligen und der Verdammten, die reiche Vielfalt der Köpfe und der Teufel und die Gesten und Stellungen der endlosen Figurenzahl. Unser Erfassen ist eher ein sinnliches, aber unser Erleben erschüttert die Seele. Vasaris Lieblingswort, um dies zum Ausdruck zu bringen, ist terribilità, die Fähigkeit, Furcht zu erregen, so gegenwärtig macht das Bild uns den Schrecken und das Erschrecken des letzten Tages der Menschheit. „Und hier ist Christus", sagt er, der, mit schrecklichem, drohendem Antlitz sich zu den Verdammten wendet und sie verflucht, und selbst unsere Liebe Frau erschrickt zutiefst und verhüllt sich in ihren Mantel, vor dem Anblick der großen Zerstörung.

Vasari hat sicher recht, wenn er seiner Beschreibung den Schrecken des jüngsten Tages zugrunde legt, auch wenn das Antlitz Christi keineswegs der faccia orribile e fiera entspricht, mit dem er es kennzeichnet und die Madonna eigentlich im Bilde nicht erschrocken erscheint, wie er sagt, sondern eher besonnen, nachdenklich, den Blick den eben jetzt Auferstehenden zugewandt, denen sie noch ihren Schutz, ihre Liebe widmen kann. Es läßt sich nicht alles auf einmal beschreiben, und was wir sehen, da die Figuren vor uns zum Leben kommen und sich bewegen, geschieht ja auch nicht in einem Augenblick. Noch steigen Auferstandene zum Himmel empor, schon fallen bereits Verdammte zur Hölle, noch kämpfen Engel und Teufel um die Rettung oder Verteufelung von Sündern, die die Gnade Gottes doch noch erlösen kann. Für die, die ihre Sünden nicht bereuen konnten, ist und muß der Anblick Gottes schreckhaft sein; sie sehen darin seine Gerechtigkeit und flüchten vor ihr in die Hölle. Dies sieht Vasari, obwohl es nicht im Bilde körperlich sichtbar ist, weil er das Geschehen im Bilde poetisch anteilnehmend miterlebt. Unendlich rührend ist die stille Trauer, mit der die Heiligen rechts zu den in die Hölle stürzenden hinab blicken und Gottes Urteil rechtfertigen. Denn die Sünder, die nicht bereuten, halten noch jetzt an ihren Sünden fest und sind dazu verdammt, sie noch einmal in ihrem Falle zu verwirklichen. Sie bestreiten die Gerechtigkeit des göttlichen Gerichts. „Ich ward gezwungen zu dem was ich tat“, ,ich war ein Opfer von Umständen“, ,,ich war arm“, ,ich war reich“, ,ich tat nur meine Pflicht!“ Der Kreuzträger wiederum, der hinab zur Hölle blickt, in Regionen, die nicht mehr im Bilde sind aber sich dahinter fortsetzen und dessen Identität von ernsten Forschern, die alles wissen müssen, umstritten ist und in dessen Anblick wir, wenn wir nicht das Bild nach Kreuzträgern absuchen wollen, um so eine endgültige Auswahl der Identität zu treffen, berührt werden, da $\beta$ er der reuige Schächer sein mag, der Christus am Kreuze neben ihm um die Vergebung seiner Sünden bat und nun, im Anblick der gerechten Verdammung der verstockten Sünder anzeigt, daß Christus dem reuigen Sünder ein Gott der Gnade ist.

Das Bild kommt diesem Ahnen entgegen; wir erwarben uns mit ihm eine lebendige Erfahrung eines Blickes, der doch in seinem beseelten Schweigen unergründlich bleibt. Und dasselbe ließe sich über die Identität des Kreuzträgers sagen. Ist er nicht der gute Schächer, so ist er ein anderer Sünder, der in seinem Erlebnis des Kreuzes sein Seelen- 
heil fand. Ist doch Petrus selbst, der jetzt voll Sorge seine Schlüssel, die er und seine Nachfolger verwalteten, hier in der Kapelle der Päpste am jüngsten Tage zurückerstattet, ein reuiger Sünder, der Christus verleugnete und daraus lernen durfte, was menschliche Schwäche und was Vergebung sei.

Das Bild, in andern Worten, bewegt uns, weil es sich vor uns bewegt, weil es, wie das Ereignis, das es darstellt, vieles, auch das undarstellbare, in einem begreift. Vasari ist der Verkünder dieses Wunders der Kunst. Er will, daß wir sie ehren, weil sie uns, wie die Poesie, ja anschaulicher noch als diese, auf unsere Herzen wirken kann, wenn wir ihr nur, das Wahre und das Gute suchend, entgegenkommen wollen. Seinen Hauptakzent aber setzte er auf die terribilità des Werkes, weil sie ja am ehesten überzeugt. Das Erlebnis des jüngsten Tages ist, wenn es wahr sein soll, schon von der Natur des Ereignisses her furchterregend. Ebenso aber muß es Liebe gegenständlich machen, und das Bild gibt wahrhaftig auch davon reiche und schöne Zeugenschaft. Nicht nur das Gericht Gottes, auch seine Gnade ist schrecklich.

Als ich vor Zeiten, noch vor der segenbringenden Restaurierung, die dem Bilde jetzt wieder zu seinem eigentlichen Aussehen verhilft, in Rom in die Capella Sistina ging, um es noch einmal zu sehen, und auch um mich bei ihm für den Versuch, den ich auch damals vornahm, zu entschuldigen, in Worte zu kleiden, was ja keiner Worte bedarf und uns doch zum Worte drängt, hatte ich das Glück, als man die Kapelle schloß, das Bild im Licht des hellen Tages zu sehen, da man die elektrische Beleuchtung abgedreht hatte, um uns zum Weggehen zu bewegen. Die Färbigkeit des Bildes nahm plötzlich zu. Die Engeln mit den Marterwerkzeugen Christi flogen nun durch blaue Luft heran, aus weiter Ferne und doch schon so nahe. Der Nimbus um Christi Haupt leuchtete auf und gab in seiner goldenen Farbe dem ganzen Bilde, ja der ganzen Kapelle eine ganz eigene Feierlichkeit und einen Zielpunkt himmlischer Ruhe und Beständigkeit. Die Szenen unten aber verschwanden in einem Halbdunkel, so daß wir sie wohl sehen konnten, aber nur wie durch einen Schleier. Bei Kerzenlicht auf dem Altar vor dem Bilde wäre alles wieder sichtbar geworden, aber im Flackerschein der geht und vergeht. Oben aber war alles hell, und erleuchtete die ganze Kapelle. Das, glaube ich, ist der richtige Auftakt zum Anblick des Bildes, den Vasari vielleicht voraussetzte, aber nicht in Worte kleidete, nicht nur weil er starke Töne brauchte, um den Höhepunkt seines Buches richtig zu kennzeichnen, sondern auch weil er überzeugen wollte, wie zu tiefst ernst das Bild war, das sobald es enthüllt wurde, ja sogar während es im Werden war, der Frivolität geziehen wurde. Beides, die Schätzung des Bildes und seine Mißachtung, ging Hand in Hand. Wesentlich an der Kritik ist, daß das Bild beschuldigt wurde, das Dekorum der päpstlichen Kapelle, ja die Heiligkeit des Gegenstandes selbst zu verletzen. Der Fokus dieses Empfindens, das, wie ich glaube, viel ernster zu nehmen ist als heutzutage üblich, ist die Darstellung der Nacktheit in einer völligen Mißachtung von Konventionen, deren wir uns, nicht unbedingt zu unserem Vorteil, entwöhnt haben. Die Beachtung derselben ist vor allem eine Geste der Mäßigung, der Bescheidenheit, der Höflichkeit, und, in dem feierlichen Rahmen kirchlicher Handlungen, im Wesen des Zeremoniells ist sie auch die verbindliche Grundlage des Gottesdienst überhaupt. Aber noch viel primärer als dies, erregt die freie Darstellung des Nackten Aufsehen und Lüsternheit. Sie war nicht ohne Grund verpönt. Schon als Gesprächsthema vor dem Bilde wirkt es fatal; und doch, fängt man einmal 
damit an, so wird das Bild vom Bilde, das ich eben für Sie zu malen versuchte, ein ganz anderes. Nicht weil wir das Bild nun falsch verstehen, sondern weil die Nacktheiten sich uns nun aufdrängen, weil sie eben da sind. Es genügt nicht, darauf hinzuweisen, daß Signorelli, dem Michelangelo in vieler Hinsicht folgte, in seiner Darstellung der Auferstehung genau so, und aus genauso guten Gründen, die Toten nackt aus leeren Gräbern steigen läßt, wie Michelangelo es tut. So viel ich weiß, da ja die Gründe, die man anführen kann, von der Natur der Sache her triftige sind, hat sich nie jemand über Signorellis Fresken beklagt. Michelangelos nackte Figuren aber sind unendlich viel lebhafter als Signorellis, da er wirklich die Kunst besaß, die Vasari so an ihm lobte, abgebildete Figuren in ihrer Ganzheit lebendig zu machen, wie Prometheus selbst, so sehen wir sie auch nicht als Teile eines Kunstwerks, sondern das Kunstwerk selbst verschwindet vor der Lebendigkeit, der Selbständigkeit ihrer Existenz. Die nackten Figuren müssen daher als fleischlich nackt von uns empfunden werden. Man spürt den Trotz, mit dem Michelangelo seine Kunst und damit auch uns auf die Probe stellt. Das Pathos des Dargestellten soll das Verfängliche, das nicht als solches zu verstehen ist, besiegen. Die Nacktheit dient dem Lebendigmachen, sie ist das Leben, und das Nichtbeachten ihrer eigenen Nacktheit ist wiederum das Zeichen ihres vollkommenden Aufgehens in dem unfaßbaren Geschehen des jüngsten Gerichts und der Wesentlichkeit himmlischer Figuren.

Schon als Michelangelo die Sixtinische Decke enthüllte, fielen seine ignudi bei allem Lob, das man ihnen als Leistungen der Kunst zollte, gelegentlich mißliebig auf. Bei dem «Jüngsten Gericht» war es unweigerlich, daß seine Nacktheiten so auffielen. Es stimmt nicht, wie man oft beschwichtigend erklärend sagt, daß Michelangelo ein Opfer einer Zeitenwende wurde, daß man später, also nach Paul III., nicht mehr verstand, was früher selbstverständlich gewesen wäre. Durch die ganze Kunstkritik zieht sich, von der Zeit der Sixtinischen Decke her bis ins späte 19. Jahrhundert, die Diskussion um das Nackte in der Kunst Michelangelos, und unumschränktes Lob seiner Kunst und Bedenken über sie erscheinen gleichzeitig und halten sich die Waage.

Die Ernsthaftigkeit des Vorwurfs ist uns fremd geworden, weil wir mehr Angst davor haben. der Pruderie geziehen zu werden, als wir das Bedürfnis empfinden, Schamhaftikeit zu ehren. Ich will hier nur andeuten, daß das sittliche Argument keineswegs so dumm oder eigennützig ist, wie man den sattsam gescholtenen, aber selten gelesenen Schmähbrief Pietro Aretinos über das «Jüngste Gericht» sich erklärt und damit mundtot macht. Nur wer vor dieser Nacktheit Scheu empfindet, kann erschauern in ihrem Anblick, wie die Wahrheitsliebe Michelangelos sie in diesem Bild der Auferstehung allen Fleisches uns vorstellt. Unsere Scheu ist die Brücke zu dem Mitleid und der Furcht, die uns vor diesem Bilde bewegen; so wie er es, hingewandt und im Hinblicken auch Wegblicken müssen in uns selbst hinein, so treten wir in das Bild ein, das uns Reue, Liebe und Gottesfurcht immer neu empfinden läßt, mit derselben Dankbarkeit für die Existenz dieses Kunstwerks, der die Kunstgeschichte Vasaris ihr höchstes Lob zollt. 


\section{Die strenge Kunstgeschichte von Moritz Thausing bis Alois Riegl}

Die Kunstgeschichte der letzten hundertundzwanzig Jahre kehrte sich mehr und mehr von dem Vorbilde Vasaris und seiner Nachahmer und Nachfolger in der Kunst, Bilder miterlebend darzustellen, ab, im Namen der Wissenschaft, die eben etwas anderes wäre, als das Kunstverständnis der Amateure in der Geschichtsbeschreibung - obgleich dieselben wohl wußten, wovon sie sprachen, da sie ja selbst vorzüglich Maler waren - leisten konnte.

Ich kann diese Epoche der neuen Kunstgeschichte, die sich selbst mit Vorliebe als die eigentliche oder wahre Kunstgeschichte vorstellt und ihre Vorgänger in eine sogenannte „Vorgeschichte der Kunstgeschichte“ abschiebt, nur an einigen Stellungnahmen zur Kunst Michelangelos zitieren, aber nicht erörtern. Sie ist uns ja wohlbekannt und ich wäre der letzte abzustreiten, was sie geleistet hat. Es ist ja auch mein Versuch, über die Geschichte des Lobes in der Kunstgeschichte nachzudenken, dem Boden der modernen Geschichtswissenschaft, die sich selbst über die geschichtlichen Voraussetzungen ihrer Auffassung der geschichtlichen Methodik befragt, entsprungen.

Wie viel Schönes und Lobenswertes wurde in diesen einhundertundzwanzig und mehr Jahren der strengen Wissenschaftlichkeit in der Kunstgeschichte entdeckt, wenn auch selten, als ob dies ein verfängliches Bemühen wäre, von ihr gepriesen. Schon zu Beginn, ja an der eigentlichen Grenzscheide der neuen Periode, sprach Moritz Thausing, dem wir die erste und noch immmer bedeutende, auf kritische Quellenstudien aufgebaute Dürermonographie verdanken, das hoffnungsfrohe Wort aus, es wäre gut, wenn man endlich eine Geschichte der Kunst schreiben könnte, in der das Wort ,schön“ gar nicht vorkäme. Als Historiker hatte er nichts übrig für ewige Werte, vermutlich mit Ausnahme derer, denen die strenge Wissenschaft sich verpflichtet fühlt, also, sich klar ausdrücken, Quellen suchen und immer von Neuem prüfen, mit Fußnoten belegen, nicht lügen oder von anderen Historikern stehlen, usw., das heißt, die historische Wissenschaft selbst, so wie sie sich im 19. Jahrhundert von der Tradition der älteren moralisch orientierten Geschichtsschreibung absonderte, zum ewigen Wert und relativierenden Prüfstein aller anderen Werte zu erheben.

Nun war aber Thausing keineswegs ein kunsthistorischer Nihilist. Er schrieb ja sein Dürerbuch, weil er in Dürers Kunst selbst Werte fand, denen er mit Hilfe seiner Wissenschaft näher kommen wollte, Werte, die er schon schätzte, bevor er sich wissenschaftlich um sie bemühte. Doch war er vorsichtig. Bemühte sich Dürer sein Leben lang um das Schöne in der Kunst, so bemühte sich Thausing um Dürer, der sich illusorisch, aber mannhaft um das Schöne bemüht hatte. Thausing war vielleicht zu seinem Glück nicht ganz konsequent in seiner Einstellung zum historischen Relativismus. Das kam später.

Wir müssen Thausing und seinen Kampfgenossen um die wissenschaftliche Kunstgeschichte auch zu Gute halten, daß sie als Historiker für die Freiheit der Kunst eintraten, die seit dem 18. Jahrhundert von der Philosophie im Namen der Ästhetik nach den von ihr vorgeblichen entdeckten unabänderlichen Gesetzen der Schönheit gerichtet und aussortiert wurde. Diese Überheblichkeit und dazu die Schwärmerei, die - ausgelöst von dem Vorbild Winckelmanns, der imstande gewesen war, haltloses Schwärmen mit wis- 
senschaftlicher Akribie zu verbinden - die Begegnung mit der Kunst der Phantasie und dem Geltungsdrange des Betrachters auslieferten, konnten im Namen der Vernunft und der geschichtlich verbürgten Wahrheit nicht unbeantwortet bleiben.

Das Lob starb nie ganz aus in der Kunstgeschichte. Es wurde nur oft verdrängt. Man lobte lieber den Künstler in der biographisch nacherzählenden würdigenden Darstellung seines Lebens und seines künstlerischen Strebens, als die Wahrheit oder Wahrscheinlichkeit dessen, was der Künstler selbst im Kunstwerk lobte, da man mit diesem selbst, wie man es auffaßte, nicht mehr in Übereinstimmung war, zumal nicht in Fragen der Religion und des Fürstenlobs. Hermann Grimm, bei all seinem feinen, das Gute suchenden Kunstverständnis, kam besser mit seiner biographisch vorgestellten Schilderung des titanischen Strebens Michelangelos aus, als mit des Künstlers Darstellung des «Jüngsten Gerichts.» Er sieht in dem Bilde des Gerichtes den Augenblick der Rache, der Vergeltung, die er, wie er sie im Anblick des Bildes erlebt, mit Recht als des Schöpfers und des gerechten Richters für unwürdig hält und erklärt sie, im Leben Michelangelos, mit Bedauern für zeitbedingt. Michelangelos Kunst wäre in diesem, seinem größten Bilde, ein Opfer seiner Zeit, der Gegenreformation, geworden. Seine Verehrung für Michelangelo selbst, den Titanen der Kunst, den Helden seiner Darstellung, wird dabei nicht beeinträchtigt, ja, im Gegenteil, da die Biographie Michelangelos tragisches Leben als Künstler und Mensch vorstellt, noch erhoben.

Doch sollte es bald auch, trotz allem Einspruch, zu einer lange anhaltenden Reaktion kommen auf diese ruhmreiche Ausflucht der Forschung, uns Kunstwerke auf der Bühne des geschichtlichen Erlebens des Künstlers, also in einer indirekten Beleuchtung des Lichts, das sie selber erschaffen, vorzustellen. Das Kunstwerk wird dabei relativiert, und der Künstler, angetan mit dem Mäntelchen, Barrett und Degen seiner Vergangenheit, historisch interessant.

Nicht im Rausche der Künstlerbiographie, die zwar die Werke des Künstlers erhellen kann, sie aber auch nur allzu oft durch den malerischen Anblick der historisch verbürgten Rahmengestaltung, der sie umgibt, ihrer eigenen Aussage entfremdet, sondern im kühlen Licht der wissenschaftlichen Analyse wollte Heinrich Wölfflin der Kunst wieder zu ihrem Rechte verhelfen, für sich selbst betrachtet zu werden. In einem Jugendwerk von 1891, das er Die Jugendwerke des Michelangelo nannte, führte er in seiner Vorrede aus, wie das Lob der Kunst Michelangelos in seiner geschichtlichen Gebundenheit an seiner Kunst vorbei gehe ohne diese zu berühren, wie vom Blick seiner Zeit aus die Jugendwerke Michelangelos neue Bedeutung erhielten, und wie er schließlich glaube, von dieser Sicht des Ursprungs seines künstlerischen Schaffens die Kunst Michelangelos sich selbst genügend in einer logisch bedingten Entwicklungskette sich fortbewege. Er sieht darin den Schlüssel zum Verständnis der Kunst Michelangelos, als reine Kunst betrachtet, unbeschwert vom stofflichen Inhalt und von der Last des Lebens. Hinter der verhaltenen, manchmal leicht ironischen Sprache Wölfflins verbirgt sich, wenn auch höflich einschränkend, eine Auffassung von dem hohen Wert der Kunst, die man am besten lobt, weil sie eben so rein ist, wenn man sie gar nicht lobt; man käme ihr sonst, so wie die Biographen, zu nahe.

Wölfflins Zurückhaltung des Lobes, ja, seine Abneigung überhaupt, im Rahmen der Forschung Lob zu spenden, zeigt doch noch ein verstecktes Lob der Sixtinischen Decke 
im Vergleich zum «Jüngsten Gericht» an, aber es tritt, soweit es sich überhaupt merken läßt, in historisierender Qualifikation auf, ,,in der Sixtina ist uns die Decke viel werthvoller als das Jüngste Gericht ...," so ist es eben bei uns, zu anderen Zeiten war es anders. Wölfflin will nicht darüber streiten, er tritt der Wahrheit wohl mit einer zeitbedingten Vorliebe gegenüber, andere taten es zu anderen Zeiten anders, jede Zeit hat ihren Zug; glaubte man früher es besser zu wissen, so galt das nur so lange man es eben konnte. Doch ist er kein sturer, sondern ein gepflegter Relativist. Über dem Zug der Zeiten steht die Kunst Michelangelos mit den ihr eigenen Stilgesetzen und Entwicklungsbedingungen. Die glaubt er mit Hilfe der Stilanalyse in ihrer absoluten Wahrheit feststellen zu können. Sie gehören der Kunst an; alles andere, und das beinhaltet die Kultur des Lobes, in der Kunst selbst wie in der Rezeption, ist Illusion der Zeiten. Wölfflin führt dies in seinem Hauptwerk, den Grundbegriffen der Kunstgeschichte, weiter aus, nicht im Argument, sondern in wohlausgesuchten Vergleichen, deren Resultate als Ewigkeitswerte vorgestellt werden. Er wünscht gar nicht, daß man diese lobt; sie sind was sie sind. Wohl aber, in dieser Sicht der Forschung, ist ihre Entdeckung lobenswert. Es ist ein Durchbruch zur wahren Erkenntnis der Kunst.

Noch zu meiner Studienzeit waren die Grundbegriffe, wenn auch schon von der Skepsis benagt, die Grundlage für die Einführung in die Kunstgeschichte, man lehrte so, Kunstwerke richtig zu sehen. Der Schüler erhielt so sein Auge, den ungetrübten Blick auf das Kunstwerk an sich. Was man aber sah, waren Kunstwerke, an denen man die Grundbegriffe herauslesen lernte; je besser das Kunstwerk die Grundbegriffe illustrierte, desto besser paßte es in seine Zeit und wenn es auch nicht dafür belobt wurde, wurde es doch ein geschätztes Schulbeispiel in dem Entwicklungsgang der Kunstgeschichte überhaupt, in der alles zur Zeit der Blüte seiner Stilperiode gleiches Recht hatte wie jede Blüte einer anderen Periode. Die klassische, glückliche und entwicklungsbedingte Höhe jeden Stils wurde geschätzt und genossen, obwohl man das nicht als Wissenschaftler zugab, sondern nur als Privatmensch, weil das Ausgewogene und Satte, die schöne Ruhe der Würde des Menschen, wenn sie in abstrakter Form erfaßt werden soll, im Gleichnis mehr entspricht als das noch im Werden Stürmische der Anfangsstile - die aber die Hoffnung auf künftige Ruhe schon in sich bergen.

Als, fast gleichzeitig mit Wölfflin, Alois Riegl den Begriff des Kunstwollens kreierte und in seiner Spätrömischen Kunstindustrie einen sogenannten Spätstil zu einem in sich ruhenden Urstil erhob, mit einem ihm eigentlichen Kunstwollen das alle Räder dieses Stils trieb, nicht nur in der Kunst, sondern auch in dem Wollen der Zeit überhaupt, wurde die Kunstgeschichte in ihrem eigenen Wirken und Wollen völlig unparteiisch und, wie sie vermeint, da sie nur historisch-wissenschaftlich aber nicht geschmacksbedingte Urteile fällt, in ihren Errungenschaften über ihre Zeit erhoben. Sie ist eigentlich in ihrem Streben nicht weit von dem von Wölfflin entfernt. Nur ihre Methoden sind verschieden, nicht aber ihr Einverständnis mit der unabänderlichen Entwicklungsgesetzlichkeit der Weltgeschichte Hegels. Beide Parteien, inbegriffen der historisierenden Ikonographie die beiden dient - und bei allen Verdiensten, deren sie sich mit Recht rühmen, blicken auf die Tradition des Lobes wie auf eine Kinderkrankheit der Kunstgeschichte zurück. Die Tradition bleibt aber weiter interessant, weil sie Indizien liefert zur Agnoszierung des Kunstwollens, zur Diagnose der Stile und Zeiten. 


\section{IV. «Das Jüngste Gericht» als verfängliches Kunstwerk: Giulio Claudio Argan und die Jesuiten}

Es ist kein Wunder, daß Versuche, Kunstwerke der alten Kunst in einer Art Kampfesstimmung zu loben, seit dem Ende des zweiten Weltkrieges mehr oder weniger verpönt sind. Statt dessen zieht man sich mehr und mehr in eine an der Soziologie orientierte, vorgeblich wertfreie, historisch total relativierende Kunstgeschichte zurück. Es gibt Zwischenstellungen, oft denkt man gar nicht darüber nach, was, obwohl Kunstwerke und schon gar die der Kunst der Moderne moralisch plädieren, an ihnen überhaupt kritisch oder lobend zu begreifen ist; es genügt, daß, wenn sie Erfolg haben, aus ihnen nachweisbare Tatsachen, also geschichtliche Werte geworden sind, die sich in einem neutralen Raster von Daten und Stilen einer Welt von Museumsbesuchern und Zeitungslesern vorstellen lassen, die dann damit nach Geschmack zurecht kommen mag wie sie will.

Die Überheblichkeit, die nicht urteilen will, weil sie Verantwortlichkeit für das gesprochene Wort selbst nur bedingt begreift, trägt, sich blähend, den Mantel der Bescheidenheit, ja der Demokratie, der alle Meinungen gleich recht sein sollen. Und doch ist das Rüstzeug dieser Ablehnung des Werturteils, der Relativierung von Lob und Tadel, nichts anderes als die wertfrei betriebene Beschreibung der Geschichte des Erfolges in der Welt, im Leben wie in der Kunst, in ihrer Entwicklung.

Ein extremer Fall, der vor einiger Zeit die Presse bewegte, die ihn wohl verflachend, aber schon deswegen einflußreich dargestellt hat, erlaubt uns vielleicht zu sehen, wie sich die Geister scheiden und was aus Lob und Tadel, also aus dem Kunsturteil selbst, in unserer Gegenwart geworden ist.

Am 16. Oktober 1992 berichtete der Messagero in Rom, daß die Jesuiten Roms in ihrem Leibblatt, der Civiltà Romana, das «Jüngste Gericht» Michelangelos aufs Korn genommen hätten. Das Werk wäre wohl als Kunstwerk bewundernswert, aber theologisch unhaltbar und dem wahren Christentum fremd. Michelangelo hätte einen richtenden Christus dargestellt, der uns die Hoffnung raubt, welche die Grundlage des Glaubens und der Versöhnung mit Gott ist: ,[ha] dipinto un Cristo che non offre speranza. "Christen sollten sich sozusagen vor dem Kunstwerk hüten.

Die Presse, nicht faul, wandte sich an den leider seither verstorbenen führenden Kunsthistoriker Italiens, Giulio Claudio Argan. Was halte er von dieser Anschuldigung? Argan, scheinbar überrascht, gab seinem Erstaunen darüber Ausdruck, daß die Jesuiten in der Zeit des heutigen Elends keine anderen Sorgen hätten, und meinte mokant, sie kritisierten das Kunstwerk ebenso wie seinerzeit der berüchtigte Pietro Aretino, der es ebenfalls, wie viele andere auch, wegen der vielen Nacktheiten für unziemlich hielt. Die neuen Jesuiten aber hätten sich anstatt über die Nacktheiten, über den Schrecken beklagt, den das Werk Gott selbst, wie sie meinten, andichtete. „Um aber ernst zu sprechen“, fuhr Argan fort, „die Kritik der Jesuiten selbst ist skandalös, nicht das Bild.“ Sie sei (ich interpretiere, indem ich zusammenziehend berichte) laienhaft, nämlich unhistorisch. Das Kunstwerk entspräche dem Geist der Zeit, in der es geschaffen wurde, ,, ' affresco esprime il proprio tempo“. Gemalt zwischen 1540 und 1545, als Rom noch an den Folgen des füchterlichen Sacco di Roma blutete und litt, zur Zeit, da die Kirche die Gegenreformation etablierte, um mit allen Mitteln gegen die Gefahr des Protestantismus aufzutreten, 
zu der Zeit, in der in Rom noch Trauer und Verwirrung herrschten, wäre das Werk folgerichtig und zeitgerecht gewesen. Er setzte dagegen das schöne, hoffnungsspendende frühere Altarbild Peruginos in der Sistina, das eben in einer noch glücklichen, noch nicht enttäuschten Periode der damals aufgehenden Renaissance gemalt worden war.

Argans zweiter Punkt war, daß der Christus des Bildes aus den bereits genannten Gründen nicht dem Neuen, sondern dem Alten Testament entsprechend dargestellt sei; harte Gerechtigkeit und nicht Gnade, wie sie eben Luther für sein Verständnis des Christentums damals in Anspruch nahm, wäre die Antwort der höchsten Hierarchie der römisch-katholischen Kirche auf Luther gewesen. „Siamo in quest'epoca e dimenticarlo mi sembra sbagliato e grottesco. Un torto inammissibile nei confronti di Michelangelo che in questa visione certo venata di pessimismo volle esprimere l'ansia di moralisazzione del suo tempo."

Giulio Claudio Argan und die Jesuiten sehen also in dem «Jüngsten Gericht» Michelangelos dasselbe, ein rachebewegtes, unerbittliches Strafgericht. Die Tradition, das Bild so zu erfassen, lebt immer wieder auf in Kritiken und gelegentlich leider auch in jubelnden Befürwortungen des Bildes. Man kann es so sehen und auch mit der Auffassung vereinbarer Texte belegen, aber auch ganz anders, wie ich an einem zweiten Beispiel, einem Aufsatz, der allerdings schon fast neunzig Jahre alt ist, also noch am Rande der Tradition der Kunstgeschichte, die lieben und loben konnte geschrieben wurde, darstellen möchte. Halten wir aber vorerst fest, daß die Jesuiten - die ja die Erklärung des Bildes von der Kunstgeschichte, die es selbst mit Vorliebe für racheschreiend hält, übernommen haben - dasselbe nach seinem absoluten, erlösungsspendenden Wert, also auf Michelangelos Lösung seiner höchsten Bildaufgabe hin befragen, während der Kunsthistoriker Argan, und mit ihm eine überwiegende Mehrzahl der Fachgenossen, Lob und Tadel bei der Beurteilung des Werkes eigentlich aus dem Wege geht und doch dabei die Jesuiten zu rügen vermag, die sich dem Bilde mit christlichem Verständnis zu nähern versuchten und das Bild tadelten, weil sie Rache sahen, wo sie mit Recht die Liebe finden wollten.

Hören wir wie Carl Justi, der zwar schon in der aufgehenden Blütezeit des historischen Relativismus in der Kunstgeschichte lebte, es aber doch, in seiner Eigenschaft als Historiker, vorzog nicht mit der Zeit zu gehen, dasselbe empfand wie die Jesuiten, aber mit mehr Vertrauen auf die große Menschlichkeit Michelangelos, die sich am Christentum und in dem Erleben der Divina Commedia Dantes gebildet hatte, das Bild ganz anders als die auch schon damals übliche Kunstgeschichte betrachtete.

Das Weltgericht hat wohl von allen seinen Werken die meisten Anfechtungen erfahren, vom Augenblick seiner Enthüllung an, sogar von seiten der unfehlbaren Auftraggeber. Die Anstößigkeit vom Gesichtspunkt des kirchlichen Decorum hätte beinahe zu seiner Zerstörung geführt. Aber ein empfindlicherer Einwand konnte von einem anderen Gesichtspunkte erhoben werden: die Religion selbst, deren Triumph es darstellen sollte und des erhabenen Stifters dieser Religion der Liebe. Der herrschende Ton ist der des Schreckens, heißt es. Dies Jüngste Gericht, so hört man, ist ,ein einziger Racheschrei.' Es hätte demnach eine Localfarbe der bedenklichsten Art. ... Hier aber überdauert die Rache Äonen - und 
den Versöhner Tod. Eine solche Gesinnung dünkt uns nicht einmal menschlich. Sie stünde sogar im Widerspruch mit dem Thema: denn Vollzug des gerechten Gesetzes ist kein Racheakt. Die Rachegefühle gehören in die Hölle. Man möchte sich die abwehrende Hand Jesu gerichtet denken gegen den zu ihm hinauf dringenden Ruf der Vergeltung. Die Ärgernisse gegen die der braghettino Remedur schaffen sollte, sind läßliche Sünden gegen die Blasphemie, die den Triumph der Religion in ein Molochfest verwandelt! Aber jene Schilderung ist eine arge Übertreibung, der umfangreichste, obere Teil ist erfüllt von Motiven ganz anderer Art - wie sein poetischer Text, der Dies Irae.

Carl Justi, . . 1909

Justi's Worte, so glaube ich, könnten die Jesuiten wieder mit Michelangelo versöhnen, aber auf das Argument von Giulio Claudio Argan, dem Justis Werk ja wohl bekannt war, sind sie klarerweise ohne Einfluß geblieben. Argan, wäre er seinem Argument treu geblieben, hätte Justi vielleicht so in seine Zeit eingeordnet wie Michelangelo in die seine, und auch sich selbst hätte er, dessen langem Wirken für die Kunstgeschichte und dem Schutz von Kunstwerken wir viel verdanken, unter denselben Schutz des Provisos gestellt, daß3 sein Leben eben seiner Zeit entsprochen hätte. Ob dies Bescheidenheit ist oder Hochmut, der sowohl Michelangelo wie Justi Befangenheit in ihrer Zeit zumißt, weil man sie auch für sich in Anspruch nimmt, wage ich nicht zu entscheiden. Vielleicht ist beides am Werk in der Doppelzüngigkeit der geistesgeschichtlichen historischen Dialektik.

\section{Epilog: Der gute Geschmack in Kunst und Leben}

Kunst und Liebe umarmen nicht was schön ist, sondern was eben dadurch schön wird.

\section{Karl Kraus}

Auswahl aus dem Werk, ed. Fischer, p. 317

Gestatten Sie, daß ich am Ende meiner Ausführungen über das Lob in der Kunstgeschichte das Andenken meines verehrten Lehrers Ulrich Middeldorf anrufe. Unter allen Kunsthistorikern unserer Zeit, die mir bekannt sind, war er der einzige, der bei Nennung des «Jüngsten Gerichts» von Michelangelo irgendwie betroffen war, ja, in einem gewissen Sinne ärgerlich wurde. Das Bild lag ihm nicht; es verletzte, so empfand er, die wesentlichen Grundlagen der Liebe zur Kunst, der er sein Leben weihte. Er sprach auch selten darüber; es war ihm peinlich. An Stelle der Klarheit, auch über höchste Dinge, wie sie uns nur die Kunst bringen kann, fand er Verwirrung; statt Größe nur Gewaltiges. Seine Vorlesungen über die Kunst der Italienischen Renaissance, in denen er die Erkenntnis des Schönen mit der des Edlen verband, beendete er am liebsten mit Michelangelos Sixtinischer Decke. So weit konnte und wollte er Michelangelo gerade noch folgen, sich an der Decke, wenn auch mit Besorgnis, erfrischen und dem Künstler dankbar sein. Wenn er auch ein moderner Kunsthistoriker war, so wollte er sich doch nicht von Unberufenen, 
auch wenn es Künstler waren, und er meinte große Künstler - denn bei kleinen kommt es ja nicht oft vor, daß sie uns verführen - leiten lassen, wohin er nicht gehen wollte; nicht aus Caprice, sondern weil er der reinen Schönheit verbunden war, in der er alles Gute, das uns zur Menschlichkeit erhebt, verankert sah.

Seine Abneigung gegenüber dem «Jüngsten Gericht» Michelangelos war also ein Geschmacksurteil. Wenn wir das Wort Geschmack hören, lächeln wir schon, und schon gar, wenn wir damit den „guten Geschmack“ meinen. Man denkt an die Bourgeoisie in gestickten Filzpantoffeln, die Thee trinkt und geeichte Kunstwerke sammelt und sie in Goldrahmen zur Schau stellt. Ob es je so eine Bourgeoisie gegeben hat, weiß ich nicht, aber selbst in der Karikatur, die zum Schlagwort - oder Schlag-Zu-Wort - geworden ist, das immer wieder bei Gesprächen über die Kunst bemüht wird, erscheint sie, gemessen an den Allüren und Spekulationen des heutigen Kunstbetriebs, fast liebenswürdig und harmlos. Goldrahmen schaden ja nicht; sie zeigen bloß an, daß uns das, was darin erscheint, teuer ist, daß wir es ehren wollen, daß es verdient, geehrt zu werden.

Für Ulrich Middeldorf war der gute Geschmack die Voraussetzung für seine Kennerschaft, in der er es verdientermaßen zu hohem Ansehen brachte. Seine Kennerschaft war sein tägliches Übungsfeld, in dem er seinen Geschmack immer von Neuem auf die Probe stellte und bildete.

Was er unter Geschmack verstand, erfahren wir am besten, wenn wir Alexander Pope's Essay on Taste lesen. Es war seine Lieblingslektüre.

True Wit is Nature to Advantage drest,

What oft was Thought, but ne'r before Exprest,

Something whose Truth convinced at Sight we find

That gives us back the Image of our Mind;

Justly to think; and readily express;

A full Conception; and brought forth with Ease.

Zeilen 327-32.

Von diesem Gesichtspunkt aus ist der Geschmack, über den es sich nicht disputieren läßt, nur der Geschmack, der auf der Zunge liegt. Der gute Geschmack kommt aus der Bildung des Herzens und der menschlichen Erfahrung, die nicht nur vom Schicksal geformt wird, sondern auch - und daher die Sehnsucht nach der schönen, nach der holden Kunst - von der eigenen Vorstellungskraft, die im steten, liebenden und doch nachdenklichen Umgang mit der Kunst unendlich an den Erfahrungen des Gegenständlichen in der Kunst bereichert wird. Ein Geschmacksurteil, das so fundiert ist, ist daher auch ein Werturteil, nicht nur in Belangen der Kunst, sondern eben auch, weil die Kunst die Natur nachahmt und bereichert, in Dingen des Lebens. Im Grunde ist es, vom Glück der schönen Künste erhellt, ein moralisches Urteil, das aber auch, schon aus Gründen des guten Geschmacks, nicht moralisiert.

Und so war es auch, wie ich glaube, die vielleicht schwerste Entscheidung Ulrich Middeldorfs äußeren Lebens, unter Hitler Deutschland zu verlassen, im ernstesten Sinne des Wortes ein Geschmacksurteil. Ich lernte ihn in Chicago kennen, wo ich drei 
Jahre nach dem Ende des Krieges meine eigentlichen kunsthistorischen Studien beginnen konnte und wo er, ich glaube seit 1938, als Professor wirkte. Er tauschte Florenz, das er liebte und wo er als Leiter der Photothek des Deutschen Kunsthistorischen Institutes eine für die Kenntnis der Kunst Italiens bedeutende Sammlertätigkeit entwickelt hatte, ein für ein übel berüchtigtes Chicago, das er nicht kannte, nicht weil er fliehen mußte, sondern weil ihm sein inneres Wesen, sein menschlich gebildeter Geschmack es ihm versagte, den Taumel mitzumachen, in den sein Amt, wie wohl die meisten öffentlichen Ämter der Zeit, ihn unweigerlich hineingezogen hätte. Schon die zum Gruß erhobene Hand, die ja nicht ohne Absicht auch wie ein Faustschlag wirkte, war ihm ein Greuel.

Als er später als Leiter des Kunsthistorischen Instituts nach Florenz zurückkehrte, baute er dort die herrliche Bibliothek auf, die in dem Hause, das er für sie fand und einrichtete, ihren Lesern Frieden, Wissen und Erleuchtung bietet, im Erlebnis einer beschwingten sprezzatura des Lernens, die man in andern Forschungsinstituten wohl nicht so bald findet. Daß er das zu leisten imstande war, verdanken wir nicht nur seiner Hingabe und unglaublichen Arbeitskraft, sondern vor allem auch seinem guten Geschmack, der wählte und ordnete, um diese schöne Freiheit des Hauses im Rahmen des Nützlichen zu fördern und zu formen.

Zweimal also griff Middeldorfs Sinn für das Schickliche, worin sich ja das höhere Geschmacksurteil vor allem bestätigt, in die Art seiner Lebensführung wesentlich ein: bei seiner Auswanderung aus der Heimat - ein Entschluß, zu dem niemand, und schon gar nicht ein Patriot alten Schlages, wie es Middeldorf war, leicht kommt - und bei seiner Rückkehr.

In seiner geschmacklichen Einstellung zu Michelangelo aber hat er sich wohl verirrt. Sic war irgendwie fehl am Platze, zu form-beengt, um dem «Jüngsten Gericht» näher zu kommen, um der Kunst Michelangelos nicht unrecht zu tun. Middeldorf wußte das wohl auch: er hat sich über das «Jüngste Gericht» nie deutlich im Vortrag ausgesprochen. Er floh geradezu vor ihm; er verspürte Gewalttätiges und wollte sich davon nicht unterjochen lassen, nicht begeistert werden, wo er nicht mitdenken konnte, wo die Urteilskraft vor der Macht der Suggestion des Werkes in Frage gestellt wurde. Aber dieselben Gründe. dieselben Voraussetzungen für das maßvolle Wesen der hohen Kunst und der Liebe zu ihr, die ihn Michelangelo-scheu machten, waren es auch, die ihn dazu führten, nicht nur Hitler im Tohuwabohu der Ereignisse zu erkennen, was vielleicht an sich keine große Kunst war - Hitlers Sprache machte es ja freien Menschen in dieser Hinsicht leicht, ganz abgesehen von dem, was er mit ihr zum Ausdruck brachte - sondern auch, aus dieser Erkenntnis, den bittern Entschluß zur Auswanderung in sich reifen zu lassen.

Nun will ich aus diesen biographischen Bemerkungen zum guten Geschmack keineswegs den Schluß ziehen, daß man Michelangelo ablehnen muß, um kein Nazi zu werden. Wohl aber glaube ich, daß wir das Kunsturteil von Kennern und Kunsthistorikern, die an Hitler Geschmack fanden, mit einer gewissen Skepsis prüfen sollten.

Meine Bemerkungen zum Andenken an Ulrich Middeldorf sollen nicht nur dem Bedürfnis dienen, meinen Dank öffenlich auszusprechen. Sie sind auch ein Versuch, in der Betrachtung eines bedeutenden Lebens, das der Kunst gewidmet war, anzudeuten, wie sehr, unter gewissen Umständen, d.h. im Rahmen eines Verständnisses der Kunst als 
schöner Kunst, moralisches Werturteil und Geschmack, und damit auch eine im besten Sinne des Wortes liebenswürdige Lebensführung, miteinander verbunden sind. Nehmen wir diese Verbindung ernst, so stellt sie auch Anforderungen an uns, denen wir gewiß nicht immer entsprechen können; aber sie läßt uns doch zumindest aufhorchen, mit dem Gefühl des dankbaren Glücks, daß es einen solchen Anspruch gibt und daß es nicht nur auf der Bühne der hohen Kunst, die sie uns geläutert vorstellt, Menschen geben kann, die sich ihm im Anblick der bittersten Not zu stellen vermögen.

\section{Zeugen und Andenken: Ein Nachwort}

Meine Damen und Herren,

Für uns alle, die mit den barbarischen Geschehnissen des 10. November 1938 bekannt sind, ist der herankommende Sonntag ein Tag des Gedenkens und der Besinnung. Er zeigt uns auch, er soll uns zeigen, daß die Wissenschaft und schon gar die Wissenschaft von der Kunst und der Geschichte ohne das Andenken an die Würde des Menschen und seiner Pflege, zu Handlangern, wenn nicht gar zu Anstiftern der Unmenschlichkeit herabsinken können. Als Zeugen und Vorkämpfer und Diener eines Andenkens und seiner Verwirklichung im eigenen Leben wie in ihrer Lehre, rufe ich an und lobe das Andenken des Historikers Edgar Rosen und des Kunsthistorikers Martin Gosebruch. Es war, es bleibt das Glück meines Lebens, daß sie meine Freunde waren. Ich vermisse die Heiterkeit unserer Freundschaft. Mögen auch Sie, in der Erinnerung an Edgar Rosen und Martin Gosebruch, die hier verbleibt und über die Vermittlung der Werke, die sie hinterließen und dem Beispiel der Kunst und Tapferkeit ihrer Lebensführung, das sie oft in schweren Zeiten gaben, auch Ihre Weggenossen werden, wie sie meine waren und auch bleiben. 